\title{
Modelo de apreçamento de opções embutidas em produtos de previdência no Brasil
}

\author{
Nicolas Soudki Saad ${ }^{\mathrm{a}, *}$, Celma de Oliveira Ribeiro ${ }^{\mathrm{b}}$ \\ a,*nicolas.saad@westernasset.com, Western Asset Management, Brasil

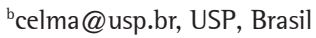

\begin{abstract}
Resumo
Este artigo apresenta um modelo para determinação do passivo e do risco financeiro associado às opções implícitas atreladas a produtos de previdência complementar no Brasil (PGBL/VGBL). Propõe-se uma modelagem específica para o problema através da caracterização da opção financeira embutida nos produtos de previdência com possibilidade de conversão do saldo final do participante em benefício vitalício sob termos predeterminados. Modelam-se também as características financeiras desse passivo, tais como duração, convexidade e fluxo de caixa equivalentes, que podem ser utilizadas para otimização da carteira de ativos associada a essa obrigação. Por fim, apresentam-se os resultados e a análise de sensibilidade da aplicação da modelagem proposta para o caso específico de um fundo de previdência complementar aberta.
\end{abstract}

Palavras-chave

Opções embutidas. Seguros. Gestão ativo/passivo. Finanças.

\section{Introdução}

A questão do financiamento de longo prazo do sistema de aposentadorias tem ganhado cada vez mais atenção em todo o mundo. A solução surgida no pós-guerra de um sistema público de aposentadorias e assistência social que se financiasse através de contribuições da massa de trabalhadores ativos foi colocada em cheque em função do envelhecimento da população global. Mesmo em países com sistema de previdência pública capitalizado como os Estados Unidos a perspectiva de envelhecimento populacional levanta a questão do sacrifício fiscal futuro que será necessário para o financiamento do sistema.

No Brasil, o sistema de previdência pública tem estado sob constante pressão financeira não somente pelo fato de se ter optado por um sistema pay as you go, como também pela sobrecarga que as demandas sociais de assistência impõem ao sistema. Além disso, para a parcela de maior renda da população, o limite máximo de contribuição/ benefício do sistema oficial se torna uma restrição extremamente relevante para financiamento da aposentadoria. Em função disso, tanto no plano global como no caso brasileiro, é essencial desenharem-se, para o longo prazo, alternativas de financiamento que complementem a aposentadoria oferecida pelo sistema oficial.

Assim, os fundos de pensão das empresas e a poupança individual voltada para a aposentadoria tornam-se os outros dois pilares do financiamento de longo prazo do sistema de aposentadorias. No caso brasileiro, os fundos de pensão já são um personagem importante do mercado de capitais, possuindo legislação específica e sendo atendidos, do ponto de vista de gestão de ativos, por um mercado altamente competitivo e eficiente. Mais recentemente, no entanto, estabeleceu-se a legislação para incentivar, através de estímulos fiscais específicos, a poupança individual de longo prazo, com a criação dos fundos denominados PGBL (Plano Gerador de Benefício Livre). Esses fundos foram estruturados de forma a permitir, através do intermédio de uma instituição seguradora, a formação de contas de poupança individualizada 
conversíveis, no futuro, em renda vitalícia sob opção do poupador. Posteriormente, a criação dos VGBL (Vida Gerador de Benefício Livre) e a modificação das regras de tributação para o participante (2005) reforçaram os incentivos para a utilização desses instrumentos para formação de poupança de longo prazo.

Nesse contexto, a indústria de previdência aberta apresentou expressivo crescimento nos últimos anos, saindo de uma arrecadação anual de R\$ 1 bilhão em 1995 para R\$ 7,7 bilhões em 2005, segundo dados da Fenaseg (Federação Nacional das Empresas de Seguros Privados e de (apitalização). Embora esse crescimento quase explosivo tenha inúmeros aspectos positivos tanto para o resultado das seguradoras quanto para a poupança de longo prazo do país, a exigência legal de se dar a opção ao participante dos planos de converter a poupança acumulada em renda vitalícia sob termos preestabelecidos cria um risco de longo prazo para a instituição seguradora.

0 objetivo deste trabalho é modelar o passivo criado e o risco financeiro associado ao mesmo quando tratado do ponto de vista da instituição seguradora. Naturalmente, a minimização e a proteção adequada desses riscos pela instituição seguradora também reduzem o risco do participante, uma vez que a primeira é um intermediário obrigatório entre o fundo (PGBL ou VGBL) e o participante.

$\mathrm{Na}$ seção 2, apresenta-se o problema detalhadamente e descrevem-se alguns modelos encontrados na literatura para opções implícitas em produtos de seguradora. Verifica-se que o caso brasileiro difere dos casos mais comuns encontrados nos Estados Unidos e no Reino Unido especialmente por se tratar de uma opção sobre uma taxa de juros a termo e não sobre um ativo de risco à vista. A seguir, é mostrada a modelagem para determinar o valor da opção concedida a cada participante e, consequentemente, o valor do passivo. São estudadas, também, as sensibilidades do passivo em relação a deslocamentos paralelos da estrutura temporal de taxas de juros. Na seção 4, encontram-se a aplicação do modelo proposto para o caso específico de um fundo de previdência complementar aberta, bem como uma análise de sensibilidade dos resultados. Por fim, apresentam-se as principais conclusões e possibilidades de desenvolvimento adicional sobre o tema.

\section{Revisão da literatura}

$\mathrm{Na}$ estrutura dos produtos de previdência em questão, a instituição seguradora não carrega os riscos financeiros durante a etapa de acumulação de poupança pelo participante, uma vez que as reservas garantidoras do benefício são definidas como o próprio saldo do participante no PGBL. Vale notar que, em contraste com a situação brasileira, em países como Estados Unidos e Reino Unido, a situação atualmente mais comum é que haja garantias de rentabilidade mínima para o segurado durante o próprio período de acumulação.

Dessa forma, Milevsky e Salisbury (2006) apresentam duas abordagens para avaliação do valor de apólices denominadas "benefício com resgate mínimo garantido" (guaranteed minimum withdrawal benefit - GMWB). Esse benefício permite ao participante a seleção da política de investimento de suas reservas, mas com a garantia de que no mínimo o valor investido the será retornado, sob condições de resgate anual máximo. São mostrados um modelo estático, sob o qual o investidor passivamente utiliza a garantia, e uma abordagem dinâmica, na qual o investidor maximiza o valor da opção otimizando o momento do encerramento da apólice.

Analogamente, Coleman, Li e Patron (2006) estudam a minimização de risco gerado pela garantia de benefício mínimo (guaranteed minimum death benefits - GMDB) associada a produtos de "anuidade variável" num mercado incompleto. Os autores concluem ser mais eficiente a utilização de opções sobre os ativos da carteira que a utilização dos próprios ativos para montagem da estratégia de proteção. No entanto, destacam a forte sensibilidade dos valores do passivo sob as causas das distribuições dos retornos dos ativos.

Schrager e Pelsser (2004) derivam uma fórmula geral de apreçamento da garantia mínima de retorno presente nas apólices denominadas unit linked, modelando as garantias como uma opção de venda sobre uma ponderação do preço do ativo de risco presente nas carteiras de investimento dos segurados.

Na situação brasileira, por outro lado, a garantia que a seguradora deve estabelecer, quando da entrada do participante no plano, corresponde às condições válidas para a conversão do saldo final do segurado em renda vitalícia. As condições predeterminadas incluem a tabela de mortalidade e a taxa de juros real que serão utilizadas para calcular o fator previdenciário, que corresponde ao fator que converte o saldo final do participante em renda vitalícia. Isso equivale a dizer que a seguradora assume uma expectativa de vida para o participante e uma taxa de juros real que serão válidas para a data de aposentadoria do participante. No entanto, nessa ocasião, o participante não tem a obrigação de converter seu saldo em renda 
vitalícia, podendo-se assumir que ele somente o fará se for financeiramente vantajoso. Essa decisão deverá levar em conta as questões de natureza tributária. Da forma tratada neste artigo, assume-se que a tributação é igual para a opção de resgate do saldo final e para a renda recebida no caso de conversão. lsso é verdadeiro para participantes que não optaram pela alteração de regime de tributação a partir de 2005. Na situação em que o participante fez a opção pelo novo regime, sua situação tributária deve ser considerada.

Dessa descrição, caracteriza-se a existência de uma opção financeira subscrita pela seguradora e adquirida pelo participante a cada aplicação adicional efetuada no plano. Como é de se esperar, a assunção da obrigação correspondente a essa opção ocorre mediante a cobrança de um "prêmio" denominado "taxa de carregamento", que consiste em um percentual do valor que o participante aporta no fundo em cada data. Diferentemente das situações mais comuns encontradas na literatura, a opção atrelada a esse tipo de garantia possui como ativo objeto um título de renda fixa, e não uma carteira composta por ativos de renda fixa e renda variável.

Lachance, Mitchell e Smetters (2003) apresentam resultados obtidos por simulação para o custo de uma opção concedida aos participantes do fundo de pensão do estado da Flórida. A opção consiste no direito de, pagando-se um prêmio, trocar-se uma vez, durante o período ativo, o benefício que será recebido após a aposentadoria. Os autores mostram que os custos da concessão da opção podem dobrar o custo do plano para o patrocinador em determinadas situações.

Kühn (2002) trata analiticamente o problema genérico de um produto em que o comprador do seguro recebe uma opção embutida, e Ballotta e Haberman (2003) analisam a situação, análoga ao caso brasileiro, em que o segurado pode optar, numa data futura, por um saldo financeiro ou uma renda vitalícia. Nesse caso, os autores modelam o direito do segurado como uma opção de compra sobre um título de renda fixa com pagamentos de cupom, sob um ambiente estocástico tanto para as taxas de juros a termo quanto para o valor do saldo do participante na conta de aposentadoria. 0 presente artigo utiliza uma abordagem semelhante àquela proposta pelos autores para determinação do valor do passivo, mas com hipóteses simplificadoras para as dinâmicas da taxa de juros e do saldo do participante. Ballotta e Haberman (2006) também desenvolvem o modelo através de simulação para tratar conjuntamente a natureza estocástica da expectativa de vida.
A modelagem do passivo correspondente às opções concedidas ao conjunto dos participantes permite a aplicação de modelos de otimização dinâmica para a definição da carteira da seguradora. Consiglio, Saunders e Zenios (2006) propõem um modelo de otimização desse tipo para a carteira de ativos e analisam a variação da carteira e do custo das apólices quando se variam as garantias mínimas de rentabilidade obtida.

\section{Modelagem}

\subsection{Valor da obrigação}

Seguindo a abordagem proposta por Ballotta e Haberman (2003), pode-se modelar o direito adquirido pelo participante como uma opção de compra sobre um título de renda fixa. Para a modelagem apresentada, serão assumidas como determinísticas as variáveis atuariais que atuam sobre o problema. Dessa forma, a idade esperada de aposentadoria do participante e a expectativa de vida deste quando da saída serão consideradas como determinísticas. Vale ressaltar que, apesar desse tratamento, não será desconsiderado o avanço das tabelas atuariais tomado desde a entrada de um participante específico no plano até a data de análise. Isso porque, ao se desenvolver o modelo de apreçamento da obrigação, será considerada uma expectativa de vida para o participante que independe daquela estabelecida em seu contrato, que será utilizada no cálculo da renda adquirida. Dessa forma, se um participante possui em seu contrato a conversão de seu saldo em renda por uma expectativa de vida de, digamos, 15 anos, mas a tabela atuarial considerada para análise indica uma expectativa de vida de 18 anos, o modelo assumirá que a renda será determinada para um período de 15 anos mas será efetivamente paga por um período de 18 anos, aumentando o valor do passivo.

0 ponto central da modelagem será, portanto, caracterizar a opção (no sentido financeiro) que é vendida ao participante na data de entrada de cada aporte realizado no fundo. Vale ressaltar que 0 termo "vender" pode ser usado precisamente, pois é comum que se cobrem taxas de carregamento para cada aporte realizado pelo participante no fundo. Mais especificamente, deseja-se apreçar a opção vendida para um participante numa certa data dado o saldo total do participante no fundo. 
Seja a seguinte notação:

- $W$ é o saldo real do participante projetado para a data da aposentadoria

- $A$ é o prazo esperado, em anos, até a aposentadoria

- $e_{c}$ é a expectativa de vida, em anos, do participante na data $A$ de acordo com a tabela atuarial preestabelecida (podendo-se incluir o tempo ao longo do qual eventuais dependentes receberão o benefício após o falecimento do segurado)

- $r_{c}$ é a taxa real contratual para conversão do saldo em renda vitalícia (discreta).

Nessas condições, a renda vitalícia mensal $R M$ será determinada, na data $A$, através da multiplicação do saldo acumulado do participante pelo fator previdenciário $F$, tal que o valor presente esperado dos pagamentos a serem realizados pela seguradora ao participante, seguindo a tabela atuarial contratada, se iguale ao saldo do participante no fundo, conforme a Equação 1:

$$
R M=F \times W \Leftrightarrow F=E_{e}\left(\sum_{i=1}^{12 \times e_{c}} \frac{1}{\left(1+r_{c}\right)^{i / 12}}\right)
$$

$\mathrm{Na}$ Equação 1, Ee corresponde ao operador valor esperado sob a variável aleatória $e c$, cuja distribuição de probabilidades é dada pela tábua atuarial. Note-se que essa operação produz um valor determinístico para $F$. Vale destacar, também, que a renda será corrigida anualmente pela variação do índice de inflação de referência, de forma que todos os fluxos de caixa e taxas de juros tratados se referem à curva de juros desse indexador.

$\mathrm{Na}$ data da aposentadoria, no entanto, a estrutura temporal da taxa de juros será definida por uma função $r_{\AA}(j): \mathrm{Z} \rightarrow \mathrm{R}^{+}$, na qual $j$ é o número de dias úteis entre a data $A$ e a data de vencimento do fluxo. Chamando de $\overline{\mathrm{e}}_{\mathrm{r}}$ a expectativa de vida do participante na data de sua aposentadoria, conforme a tabela mais adequada, o valor da renda vitalícia que o participante tem o direito de adquirir $\left(S_{A}\right)$ será dado por:

$S_{A}=\sum_{i=1}^{12 \times \bar{e}_{r}} \frac{R M}{\left(1+r_{A}\left(j_{i}\right)\right)^{j_{i} / 252}}$

Nessa relação, $j_{i}$ representa o número de dias úteis entre a data $A$ e o $i$-ésimo pagamento mensal da renda vitalícia. Portanto, comparando-se (1) com (2), deve-se esperar que um participante racional, sob uma situação de indiferença fiscal entre as alternativas, converterá sua poupança em renda somente se $\mathrm{S}_{A}>W$, pois, caso contrário, o seu saldo acumulado $W$ seria capaz de adquirir, às taxas de mercado, um contrato de renda vitalícia $R M^{\prime}$ tal que $R M^{\prime}>R M$. É razoável supor, da mesma forma, que, se $\mathrm{S}_{A}<W$, o participante converterá seu saldo em renda vitalícia.

Sob essas hipóteses, tem-se que, na data $A$, o participante $k$ terá o direito de escolher entre o maior valor entre $S_{A}$ e $W$, e, portanto, o passivo correspondente da seguradora $\left(I_{k}\right)$ será de:

$$
l_{k, A}=\max \left(S_{k, A}-W_{k}, 0\right)
$$

Entendendo-se o fluxo de pagamentos da Equação 2 como um título de renda fixa, a Equação 3 caracteriza que o ativo financeiro que o participante possui corresponde a uma opção de compra, com vencimento na data $A$, preço de exercício $W$ e cujo ativo objeto é o ativo com os fluxos da Equação 2. 0 preço hoje desse ativo será denominado $S_{k}$.

\subsection{Modelo de apreçamento e ajustes}

Como se pode observar, através da modelagem proposta até aqui, seria possível calcular-se o prêmio e as sensibilidades da opção desde que se defina um modelo adequado e se façam as adaptações necessárias.

Lee e Stock (2000) apresentam uma revisão de modelos que assumem um processo estocástico com reversão à média para a taxa de juros. No caso brasileiro, a hipótese de reversão à média pode ser muito forte, uma vez que não se pode assumir que as taxas de juros reais atuais são válidas para o longo prazo. Além disso, impor uma média arbitrária para o processo da taxa de juros introduz uma forte dependência dos resultados em relação a esse parâmetro. Dessa forma, preferiu-se trabalhar com um modelo log-normal para o preço do ativo. Kolb (2000) apresenta o modelo de Fisher-Black como adequado para apreçamento de opções sobre títulos de renda fixa (bonds), sob a hipótese implícita de distribuição log-normal para os retornos do ativo objeto. Ainda conforme Kolb (2000), o modelo de Fisher simplifica o modelo de Merton (desenvolvido para apreçamento de opções sobre ativos com pagamento de dividendos) para apreçamento de opções sobre preços futuros. A conveniência desse modelo para ativos de renda fixa é que a expressão matemática é bastante simplificada pela equivalência entre a taxa livre de risco e a taxa esperada de crescimento do preço do título. Especificamente, com a notação introduzida até aqui, a opção concedida ao participante possui o seguinte valor, segundo o modelo de Fisher: 
$c^{F}(R, A, F, W, \sigma)=c^{F}=e^{-R A}\left[F N\left(d_{1}^{F}\right)-W N\left(d_{2}^{F}\right)\right]$

$d_{1}^{F}=\frac{\ln \left(\frac{F}{W}\right)+0.5 \sigma^{2} A}{\sigma \sqrt{A}}$

$d_{2}^{F}=d_{1}^{F}-\sigma \sqrt{A}$

Em (4), tem-se:

- $c^{\mathrm{F}}$ é o preço da opção de compra pelo modelo de Fisher Black

- $A$ é o prazo da opção

- $R$ é a taxa de juros contínua livre de risco para o prazo $A$; utilizou-se $R$ como notação para a taxa contínua e $r$ como notação da taxa discreta, sendo $R=\ln (1+r)$

- $F$ é o preço a termo do ativo objeto

- $W$ é o preço de exercício da opção

- $N(x)$ é o valor da distribuição normal padrão acumulada para o valor $x$

- $\sigma$ é a volatilidade do preço a termo; por argumentos de arbitragem (KOLB, 2000), pode-se mostrar que o preço a termo é equivalente ao preço resultante do investimento no ativo à vista mas integralmente financiado pela emissão de um título de renda fixa cupom zero com vencimento coincidente com o vencimento do contrato a termo.

Cabe, no entanto, fazer um ajuste ao preço da obrigação dado pelo modelo acima. lsso ocorre porque, sob a hipótese de distribuição log-normal, o preço do ativo objeto apresenta conceitualmente um limite inferior (igual a zero), mas não apresenta um limite superior. No entanto, para o caso estudado, é de fundamental importância reconhecer que existe também um limite superior para o preço do ativo a termo, que vem a ser o preço obtido na situação de taxa de juros real igual a zero. Black (1995) argumenta que a taxa de juros nominal possui características de opção por não assumir valores negativos, e propõe o ajuste das árvores binomiais para esse fenômeno, especialmente relevante em períodos de taxa de juros baixa. Na mesma linha, propõe-se que a taxa de juros real de longo prazo tem essa característica, mas, ao invés de ajustar as hipóteses do modelo de apreçamento, assume-se que, juntamente à obrigação assumida perante o participante, a seguradora possui o direito de adquirir o fluxo ao preço dado por uma taxa de juros real nula (para uma discussão sobre o limite inferior para a taxa de juros real, ver Black (1995)). Ou seja, atrelada à opção descrita anteriormente, a seguradora possui uma posição comprada em uma outra opção de compra com as mesmas características exceto o preço de exercício $X$, que será a soma algébrica das parcelas mensais da renda do participante (pois a taxa de juros é nula):

$X=12 \times \bar{e}_{r} \times F \times W$

Com esse ajuste, chega-se à formulação final para o valor da obrigação assumida pela seguradora para com um participante específico $k$ :

$l_{k}=c^{F}\left(r_{A_{k}}, A_{k}, F_{k}, W_{k}, \sigma_{k}\right)-c^{F}\left(r_{A_{k}}, A_{k}, F_{k}, X_{k}, \sigma_{k}\right)$

Cabe destacar que, como $W_{k} \geq X_{k}$, o valor da opção com exercício $W_{k}$ será sempre superior ao valor da opção com exercício $X_{k}$, e, como era de se esperar, a obrigação $I_{k}$ assumida com o participante $k$ sempre terá valor não negativo.

0 valor da obrigação total da seguradora e dessa obrigação total como percentual do patrimônio dos fundos administrados são dados, respectivamente, por (7) e (8):

$L=\sum_{k=1}^{U} l_{k}$

$L \%=\frac{\sum_{k=1}^{U} l_{k}}{\sum_{k=1}^{U} P_{k}} \times 100$

Faz-se necessário, portanto, determinar os parâmetros do ativo objeto necessários para avaliação de (4), ou seja, o preço atual, o preço a termo e a volatilidade do ativo objeto.

\subsection{Ativo objeto da opção (preço à vista, preço a termo e volatilidade)}

0 preço à vista do ativo objeto que possui os fluxos da Equação 2 é dado por:

$S=\sum_{i=1}^{12 \times \bar{e}_{r}} \frac{F \times W}{\left(1+r\left(j_{i}\right)\right)^{A+i / 12}}$

Em (9), S é o preço à vista do ativo objeto, $r(j): \mathrm{Z} \rightarrow \mathrm{R}^{+}$é a estrutura temporal da taxa de juros indexada à inflação na data de hoje, e $j_{i}$ é o número de dias úteis entre a data atual e a data de vencimento do fluxo. Por arbitragem, o preço a termo do ativo objeto será dado por:

$F=S \times\left(1+r\left(j_{A}\right)\right)^{A}$

Em (10), tem-se:

- Sé o preço à vista do ativo objeto

- $A$ é o tempo esperado, em anos, até a aposentadoria do participante

- Fé o preço a termo do ativo objeto (para a data $A$ ) 
Para se determinar $W$ a partir de um saldo inicial $P$, é necessária uma hipótese de como esse saldo evoluirá ao longo do tempo até a data da aposentadoria. Vale destacar que, conforme mencionado, o objetivo deste trabalho é apreçar a opção embutida no saldo que o participante já possui no fundo, de forma que não será estudada a dinâmica de aportes adicionais. Para um fundo de renda fixa e sob as premissas colocadas, pode-se modelar o valor do saldo real do participante na data da aposentadoria como:

$$
W=P \times\left(\frac{1+r\left(j_{A}\right)}{1+t a}\right)^{A}
$$

Em (11), ta é a taxa de administração anual cobrada do fundo.

Para determinação da volatilidade do preço a termo, é necessário utilizar uma modelagem para a matriz de covariâncias entre os vértices da curva de juros reais (JORION, 1998). Determinam-se, inicialmente, os prazos-chave a serem utilizados para parametrização da volatilidade. Por exemplo, poderiam ser utilizados como prazos-chave os períodos de 1 dia útil, 5 dias úteis, 21 dias úteis, 156 dias úteis, 252 dias úteis, $2 \times 252$ dias úteis, $5 \times 252$ dias úteis, $10 \times 252$ dias úteis, $20 \times 252$ dias úteis, $30 \times 252$ dias úteis e $50 \times 252$ dias úteis. Determinam-se, então, por métodos específicos (JORION, 1998), as volatilidades dos retornos anuais de investimentos de valor presente unitário em cada prazo-chave, bem como a matriz de correlações entre os retornos anuais desses investimentos. Para o exemplo dado, a matriz de correlações tem dimensão $11 \times 11$, pois há 11 prazos-chave. Num passo seguinte, cada fluxo de caixa (em valor presente) é transformado (através de uma decomposição pro rata temporis) em uma composição percentual de dois fluxos que ocorrem nas datas-chave, ou seja, cada fluxo é decomposto em um vetor $p$ correspondente a uma carteira de fluxos que ocorrem nas datas-chave. A volatilidade desse fluxo é determinada, então, por:

$\sigma_{f}=\sqrt{p^{T} s C s^{T} p}$

Em (12), tem-se:

- $\sigma_{f}$ é a volatilidade do retorno do preço do fluxo específico

- $p$ é o vetor de exposições do fluxo aos prazos-chave

- $s$ é o vetor de volatilidades dos retornos dos fluxos com vencimento nos prazos-chave

- $C$ é a matriz de correlações entre os retornos dos fluxos com vencimento nos prazos-chave
Pelos argumentos de preços lineares (LUEMBERGER, 1998), pode-se ver que, se um fluxo pode ser decomposto em um vetor de exposições $p$, então um conjunto de fluxos pode ser decomposto num outro vetor $p$, e pode-se determinar, através de (12), a volatilidade desse conjunto de fluxos.

Portanto, para um fluxo específico associado a um participante, é possível não somente descrever o ativo objeto da opção concedida ao participante como também calcular seu preço (à vista e a termo) e sua volatilidade. Analogamente, é possivel calcular a volatilidade do preço a termo do fluxo, desenhando-se uma carteira com exposição 100\% ao fluxo de caixa de (2) e exposição -100\% a um fluxo de caixa igual ao preço a termo para a data $A$. A relação descrita acima é observada quando se desenvolve algebricamente a seguinte relação:

$$
\begin{aligned}
& F=S \times e^{r A}=S \times\left(e^{-r A}\right)^{-1}=S \times Z_{A}^{-1} \Rightarrow \\
& \Rightarrow \ln F=\ln S-\ln Z_{A} \\
& \sigma_{F}^{2}=\operatorname{Var}(\ln F)=\operatorname{Var}\left(\ln S-\ln Z_{A}\right)= \\
& =\operatorname{Var}(\ln S)+\operatorname{Var}\left(\ln Z_{A}\right)-2 \times \operatorname{Cov}\left(\ln S, \ln Z_{A}\right)
\end{aligned}
$$

Conforme (13), a volatilidade do preço a termo é igual à volatilidade de uma carteira de valor zero com exposição unitária ao ativo objeto e exposição de mesmo valor presente, mas em sinal oposto ao título de renda fixa sem amortização (zero coupon) com vencimento na data $A$. Essa é a justificativa para se calcular a volatilidade do preço a termo como a volatilidade da carteira descrita anteriormente.

\subsection{Sensibilidades do valor da obrigação}

Tendo sido determinado o valor da obrigação associada ao direito dos participantes, passa-se à análise de como esse valor varia em relação às variáveis de mercado. Em especial, é interessante determinar o quanto varia a obrigação total da seguradora conforme variam as estruturas temporais de taxas de juros, ou seja, os preços dos ativos objeto aos quais se referem cada opção.

Em decorrência da linearidade da Equação 7, o interesse passa a ser a determinação da sensibilidade da obrigação com cada participante $l_{k}$ em relação ao preço do ativo objeto da opção para aquele participante $\left(S_{k}\right)$.

Pelo modelo de Fisher (KOLB, 2000), é possivel calcular a derivada de $I_{k}$ em relação ao preço a termo do ativo objeto $F_{k}$, conforme (14). Nessa equação, $\Delta^{\mathrm{F}}$ é o delta da opção (primeira derivada em relação ao preço a termo) calculado pelo modelo de Fisher. 


$$
\begin{aligned}
& \Delta_{k}=\partial / \partial F_{k} l_{k}=\partial / \partial F_{k}\left[\begin{array}{c}
c^{F}\left(R_{A_{k}}, A_{k}, F_{k}, W_{k}, \sigma_{k}\right) \\
-c^{F}\left(R_{A_{k}}, A_{k}, F_{k}, X_{k}, \sigma_{k}\right)
\end{array}\right]= \\
& =\Delta^{F}\left(R_{A_{k}}, A_{k}, F_{k}, W_{k}, \sigma_{k}\right)-\Delta^{F}\left(R_{A_{k}}, A_{k}, F_{k}, X_{k}, \sigma_{k}\right) \\
& \Delta^{F}=e^{-R_{A_{k}} \times A} N\left(d_{1}^{F}\right)
\end{aligned}
$$

No entanto, de (10), sabemos que:

$$
\partial / \partial S_{k} F_{k}=\partial / \partial S_{k}\left(S_{k} \times\left(1+r\left(j_{A_{k}}\right)\right)^{A_{k}}\right)=\left(1+r\left(j_{A_{k}}\right)\right)^{A_{k}}=F_{k} / S_{k}
$$

Portanto,

$$
\begin{aligned}
& \Delta_{k}=\partial / \partial S_{k} l_{k}=\partial / \partial F_{k} l_{k} \times \partial / \partial S_{k} F_{k}= \\
& =e^{R_{A_{k}} \times A} \times\left[\Delta^{F}\left(i_{I, t_{k}}, t_{k}, F_{k}, w_{k}, \sigma_{k}\right)-\Delta^{F}\left(i_{I, t_{k}}, t_{k}, F_{k}, X_{k}, \sigma_{k}\right)\right]
\end{aligned}
$$

Portanto, para uma variação positiva/negativa unitária no preço à vista do ativo objeto $k$, o valor da obrigação aumenta/diminui em $\Delta_{k}$. Vale notar também que, analogamente à Equação 6, o delta da opção concedida é maior ou igual ao delta da opção detida, o que leva $\Delta_{k}$ a ser sempre não negativo, ou seja, a exposição delta equivalente da seguradora corresponde a uma posição de venda (pois trata-se de um passivo) num determinado título de renda fixa.

No entanto, ao agregar a sensibilidade de cada obrigação $I_{k}$ para determinar a variabilidade do passivo total em relação à variação dos preços de mercado, é conveniente utilizar-se uma medida comum de sensibilidade dos preços de cada ativo objeto $S_{k}$ às taxas de mercado. Para isso, calcula-se a derivada do valor total do passivo $L$ em relação ao patamar da taxa de juros livre de risco, definindo-se, dessa forma, a sensibilidade do valor de $L$ a deslocamentos paralelos da estrutura temporal da taxa de juros:

$$
D=\partial / \partial i=\partial / \partial i\left(\sum_{k=1}^{U} l_{k}\right)=\sum_{k=1}^{U} \partial / \partial P_{k} l_{k} \times \partial / \partial i P_{k}=\sum_{k=1}^{U} \Delta_{k} \times d_{k}
$$

Na Equação 17, tem-se:

- $D$ é a sensibilidade do valor de $L$ a deslocamentos paralelos da estrutura temporal da taxa de juros, ou seja, é a duração financeira do passivo $L$. Duração ou duration é a primeira derivada do preço do ativo em relação à taxa interna de retorno, que equivale à primeira derivada em relação a deslocamentos paralelos da estrutura temporal de taxas de juros (FABOZZI, 2000). Denominou-se aqui duração financeira a duração quando apresentada na unidade monetária (DAHL; MEERAUS; ZENIOS, 1993a).

- $d_{k}$ é a duração do ativo objeto da opção para o participante $k$

É conveniente, também, calcular-se a segunda derivada do valor do passivo $L$ em relação a deslocamentos paralelos na estrutura temporal de taxas de juros (convexidade). Analogamente à Equação 17, tem-se:

$C=\partial / \partial i D=\partial / \partial i\left(\sum_{k=1}^{U} \Delta_{k} \times d_{k}\right)=\sum_{k=1}^{U} \partial / \partial i\left(\Delta_{k} \times d_{k}\right)=$

$=\sum_{k=1}^{U}\left[\partial / \partial i\left(\Delta_{k}\right) \times d_{k}+\partial / \partial i\left(d_{k}\right) \times \Delta_{k}\right]=$

$=\sum_{k=1}^{U}\left[\left(\partial / \partial S_{k} \Delta_{k} \times \partial / \partial i S_{k}\right) \times d_{k}+\partial / \partial i\left(d_{k}\right) \times \Delta_{k}\right]=$

$=\sum_{k=1}^{U}\left[\left(\gamma_{k} \times d_{k}\right) \times d_{k}+c_{k} \times \Delta_{k}\right]=$

$=\sum_{k=1}^{U}\left[\gamma_{k} \times d_{k}^{2}+c_{k} \times \Delta_{k}\right]$

Em (18), tem-se:

- Cé a convexidade financeira do valor de $L$;

- $\gamma_{k}$ é segunda derivada da obrigação para com o participante $k \mathrm{em}$ relação ao preço do ativo objeto $k$. Ele é, portanto, o gama das opções que compõem a Equação 7. Utilizando-se o modelo de Merton pode-se calcular mais facilmente 0 gama, que, conforme Kolb (2000), é dado por

$$
\frac{N \cdot\left(d_{1}^{M}\right) e^{-r t}}{S \sigma \sqrt{t}} \text {, onde } d_{1}^{M}=\frac{\ln \left(\frac{S_{t}}{X}\right)+0.5 \sigma^{2} t}{\sigma \sqrt{t}} ;
$$

- $c_{k}$ é a convexidade do do ativo objeto da opção para o participante $k$

As Equações 17 e 18 fornecem, portanto, a duração $D$ e a convexidade $C$ do passivo da seguradora atrelado ao patrimônio total dos planos. Com essa formulação, é possível fazer um primeiro exercício de montagem de uma carteira de títulos indexados que proteja a seguradora de variações no preço dos ativos. Essa carteira é gerada através de modelos de imunização, conforme apresentados em Saad e Ribeiro (2004), e correspondem à solução do seguinte problema de otimização:

$\min _{x_{i}} \sum_{i} P_{i} \cdot x_{i}$

s.a.:

$\sum k_{i} \cdot x_{i}=k_{L}$

$\sum_{i} Q_{i} \cdot x_{i} \geq Q_{L}$

$x_{i} \geq 0, \forall i$

- $P_{i}$ é o preço do ativo $i$;

- $k_{i}$ é o duração financeira do ativo $i$;

- $\mathrm{k}_{L}$ é a duração financeira do passivo;

- $Q_{j}$ é o convexidade financeira do ativo $i$;

- $\mathrm{Q}_{L}$ é o convexidade financeira do passivo;

- $x_{i}$ é a quantidade do ativo $i$, ou seja, o valor a ser investido no ativo $i$ dividido pelo seu preço. 


\subsection{Carteira de proteção pelo delta (delta hedge) e modelo de dedicação}

A otimização apresentada em (19), embora represente uma aproximação da carteira de proteção ótima, pode ser considerada uma solução restrita para o problema, uma vez que leva em conta apenas três características agregadas do passivo (valor, duração e convexidade). É de se esperar que uma carteira de proteção mais completa leve em conta também a distribuição dos fluxos implícitos no passivo da seguradora. 0 modelo de dedicação, que otimiza os descasamentos entre fluxos de caixa dos ativos e dos passivos (SAAD; RIBEIRO, 2004), seguindo Dahl, Meeraus e Zenios (1993b), se apresenta como uma alternativa mais abrangente para a solução do problema. Sua formulação matemática é apresentada a seguir.

$$
\begin{aligned}
& \min \sum_{i} P_{i} x_{i}+s_{0} \\
& \text { s.a.: } \\
& \sum_{i} D_{i \tau} x_{i}+s_{\tau-1} \times(1+\rho)^{\Delta \tau}=L_{\tau}+s_{\tau}, \forall \tau \\
& D_{i \tau}=\sum_{t \in[\tau-1, \tau]} C_{i t} \times(1+\rho)^{\tau-t} \\
& s_{\tau} \geq 0, \forall \tau \\
& x_{i} \geq 0, \forall i \\
& \text { - } P_{i} \text { é o preço do ativo } i ;
\end{aligned}
$$

- $\mathrm{x}_{i}$ é a quantidade do ativo $i$, ou seja, o valor a ser investido no ativo $i$ dividido pelo seu preço;

- $\tau$ é o índice que representa as datas com vencimentos do passivo;

- $\Delta \tau$ é o período transcorrido entre as datas de vencimento $\tau-1$ e $\tau$;

- $\mathrm{L}_{\tau}$ é o pagamento do passivo na data $\tau$;

- $\mathrm{S}_{\tau}$ é o superávit (excesso de caixa na data $\tau$ );

- $\rho$ é a taxa de reinvestimento (que pode ser uma função do tempo);

- $\mathrm{C}_{i t}$ é o fluxo de caixa do ativo $i$ na data $t$;

- $\mathrm{D}_{i \tau}$ é o valor do fluxo de caixa do ativo ireinvestido à taxa $\rho$ entre as datas $\tau-1$ e $\tau$.

0 modelo em (20) pode ainda ser adaptado (SAAD; RIBEIRO, 2005) para tratar adequadamente os diferentes níveis de risco que diferentes indexadores trazem para os ativos (Selic, IPCA, IGP-M etc.) e para o passivo.

Para aplicação do modelo de dedicação a esse caso é necessário, no entanto, determinarem-se os fluxos de caixa do passivo equivalente à exposição criada pelas opções. Para isso, será considerado que o passivo é composto da soma dos fluxos de caixa dos ativos objeto correspondentes à obrigação para com cada participante $k$ ajustada pela exposição delta equivalente àquele ativo. Em outras palavras, como o passivo correspondente a um participante $I_{k}$ possui uma sensibilidade ao valor do ativo $S_{k}$ dada por (17), ou seja, dada pela variável $\Delta_{k}$, esse passivo corresponde a uma posição financeira vendida no ativo $S_{k}$ igual a $\Delta_{k} \times \mathrm{S}_{k}$.

Portanto, agregando-se os fluxos gerados pela posição delta equivalente correspondente a cada participante, chega-se aos fluxos de caixa do passivo $\mathrm{L}_{\tau}$, necessários para a implementação do modelo em (20).

Vale destacar, no entanto, que, diferentemente da situação ideal para o qual o modelo de dedicação é construído, na situação em questão o passivo é dinâmico, ou seja, as variáveis necessárias para implementação dos modelos (19) e (20) podem oscilar ao longo do tempo. Essa oscilação deve ser estudada de forma a se determinar se os custos de transação mais elevados são compensados pela sofisticação adicional do modelo. Portanto, é adequada uma análise de sensibilidade dos parâmetros calculados previamente à implementação dos modelos.

\section{Aplicação}

0 modelo apresentado na seção 3 pode ser diretamente aplicado a uma situação em que se tenham os dados suficientes em relação aos participantes de um plano. Na aplicação apresentada, foram tomados dados de todos os participantes vinculados a um fundo PGBL com perfil de investimento de renda fixa. Por questões de sigilo, apresentam-se valores arbitrariamente proporcionais aos valores reais para as variáveis.

\subsection{Levantamento de dados}

A área atuarial da seguradora que comercializa o fundo analisado forneceu os dados necessários à realização do estudo em questão. Especificamente, foram levantados, para cada participante com saldo no fundo, as seguintes informações:

- 0 saldo do participante no fundo;

- A idade do participante;

- A data esperada de aposentadoria;

- A taxa de juros predeterminada para conversão do saldo do participante em renda;

- A expectativa de vida do participante na data da aposentadoria, de acordo com a tábua atuarial predeterminada para o participante;

- A expectativa de vida do participante na data da aposentadoria, de acordo com a tábua atuarial atualizada. 


\subsection{Estimativa do valor do passivo}

Conforme (1), calculou-se o fator que converte o saldo do participante $W$ em uma renda mensal $R$. Para cada participante, determinaram-se também os preços à vista e a termo do ativo objeto da opção através, respectivamente, das Equações 9 e 10. Através de (11), foi determinado o saldo esperado do participante na data de sua aposentadoria. Por fim, a volatilidade do preço do ativo objeto foi calculada conforme o procedimento apresentado anteriormente e aplicando-se (12).

Tendo esses dados, foi possível calcular o valor da opção concedida para cada participante (6), bem como o valor total do passivo. A Tabela 1 apresenta as estatísticas para o valor individual da opção. As médias apresentadas não são ponderadas pelo saldo do participante.

Agregando-se os valores dos passivos individuais, chega-se, conforme (7), a um valor total para o passivo de $\mathrm{R} \$ 4,27$ milhões, ou $0,81 \%$ do saldo total dos participantes no fundo. É interessante notar que, para a condição específica do plano em questão, o valor financeiro da obrigação assumida é razoavelmente baixo, sendo compensado pelas taxas de carregamento praticadas. No entanto, esses valores são bastante sensíveis aos dados específicos do problema, especialmente no que se refere à taxa de juros média. Portanto, para planos com diferentes perfis de participante, o valor total do passivo poderia ser maior. Além disso, vale destacar, conforme será detalhado na seção 4.3, que a duração financeira do passivo é relativamente alta quando comparada com o valor do mesmo, de forma que a proteção dos riscos em relação a variações nas taxas de juros é recomendável.

\subsection{Duração e convexidade do passivo}

Conforme as relações (17) e (18), é possível calcular a duração e a convexidade do valor calculado para o passivo, ou seja, a primeira e segunda derivadas do valor financeiro do passivo em relação a deslocamentos paralelos da estrutura temporal de taxas de juros.

A Tabela 2 apresenta as estatísticas dos valores necessários para a determinação da duração e convexidade do ativo, ou seja, o valor atual do ativo objeto, a duração e convexidade financeiras do ativo objeto e as regras referentes a cada opção.

Dessa forma, calculam-se a contribuição do passivo referente a cada participante para a duração e a convexidade do passivo, indicados na Tabela 3.
Esses valores levam a uma duração financeira total do passivo de $R \$ 5,46$ milhões e a uma convexidade financeira total do passivo de -R\$ 2,19 milhões. Cabe notar que, por ser o passivo uma posição vendida em opções de compra, a duração e convexidade delta equivalentes possuem sinal oposto às durações e convexidades dos ativos objeto referentes a cada participante individualmente.

\subsection{Fluxo de caixa do passivo "delta- equivalente"}

Pode-se estimar, também, conforme esquematizado na seção 3.5 , o passivo delta equivalente correspondente ao conjunto dos participantes em análise. 0 gráfico da Figura 1 apresenta o fluxo de caixa real ano a ano assim determinado.

Esse passivo possui um valor presente total de $R$ \$ 21,7 milhões, uma duração financeira de $\mathrm{R} \$ 5,40$ milhões e uma convexidade financeira de $\mathrm{R} \$-1,55$ milhão. É interessante notar que a duração e convexidade se assemelham àquelas calculadas no

Tabela 1. Estatísticas para o valor individual da opção.

\begin{tabular}{ccc}
\hline & Média & $\begin{array}{c}\text { Desvio } \\
\text { padrão }\end{array}$ \\
\hline Valor da opção concedida (R\$) & 1.518 & 5.345 \\
Valor em percentual do saldo atual (\%) & 1,6 & 3,4 \\
\hline
\end{tabular}

Tabela 2. Estatísticas para as variáveis necessárias para cálculo do valor da opção.

\begin{tabular}{ccc}
\hline & Média & $\begin{array}{c}\text { Desvio } \\
\text { padrão }\end{array}$ \\
\hline $\begin{array}{c}\text { Preço do ativo objeto (R\$) } \\
\text { Duração financeira do ativo }\end{array}$ & 112.862 & 229.830 \\
$\begin{array}{c}\text { (para 1\% de variação na taxa) (R\$) } \\
\text { Convexidade financeira do ativo }\end{array}$ & -21.978 & 42.878 \\
$\begin{array}{c}\text { (para 1\% } 0^{2} \text { de variação na taxa) (R\$) } \\
\text { Delta da opção (\%) }\end{array}$ & 5.565 & 10.677 \\
Gama da opção (\%) & 8,1 & 11,8 \\
\hline
\end{tabular}

Tabela 3. Estatísticas para contribuição para duração e convexidade do passivo.

\begin{tabular}{ccc}
\hline & Média & $\begin{array}{c}\text { Desvio } \\
\text { padrão }\end{array}$ \\
\hline $\begin{array}{c}\text { Contribuição para a duração } \\
\text { financeira do passivo (R\$) }\end{array}$ & 1.942 & 6.148 \\
$\begin{array}{c}\text { Contribuição para a convexidade } \\
\text { financeira do passivo }(\mathrm{R} \$)\end{array}$ & -780 & 2.247 \\
\hline
\end{tabular}


item 4.3. No entanto, por se tratar do valor delta equivalente do ativo objeto e não mais do valor da opção, o montante financeiro é significativamente superior ao prêmio calculado anteriormente.

\subsection{Análises de sensibilidade}

Conforme mencionado, para a aplicação de modelos de otimização cuja solução seja robusta, é importante verificar a variabilidade dos parâmetros calculados conforme mudam os dados do problema. Para isso, foram recalculados o valor do passivo, a duração financeira e a convexidade financeira, variando-se a volatilidade e o patamar da estrutura a termo de taxas de juros. Vale destacar que, nesse último caso, o deslocamento da estrutura a termo provoca um recálculo da volatilidade para cada

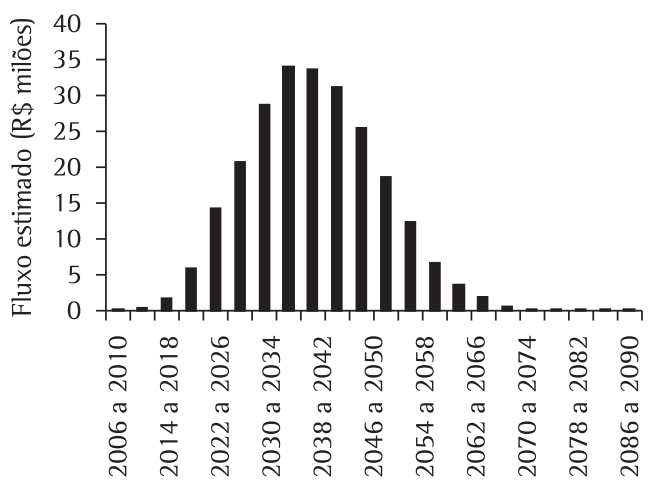

Figura 1. Passivo real delta equivalente.

Tabela 4. Análise de sensibilidade em relação à volatilidade.

\begin{tabular}{cccc}
\hline $\begin{array}{c}\text { Variação na } \\
\text { volatilidade } \\
\text { (\%) }\end{array}$ & $\begin{array}{c}\text { Valor do } \\
\text { passivo } \\
\text { (R\$ milhões) }\end{array}$ & $\begin{array}{c}\text { Duração } \\
\text { financeira } \\
\text { (R\$ milhões) }\end{array}$ & $\begin{array}{c}\text { Convexidade } \\
\text { financeira } \\
\text { (R\$ milhões) }\end{array}$ \\
\hline+20 & 5,68 & 6,30 & $-2,48$ \\
+10 & 4,97 & 5,87 & $-2,34$ \\
0 & 4,27 & 5,46 & $-2,19$ \\
-10 & 3,57 & 4,90 & $-2,03$ \\
-20 & 2,90 & 4,35 & $-1,84$ \\
\hline
\end{tabular}

opção. No entanto, não se recalculou o saldo futuro do participante conforme (11), uma vez que o objetivo da análise é determinar o efeito da variação das taxas sobre o passivo da seguradora pelo ponto de vista da opção. Para se considerar os comovimentos entre o valor da opção e o valor do investimento, é necessário um refinamento prévio do modelo.

Especificamente, variou-se a volatilidade de cada opção em $+20,+10,-10$ e $-20 \%$. No que se refere à taxa de juros, testaram-se deslocamentos paralelos de $+2,+1,-1,-2,-3$ e -4 pontos percentuais.

As Tabelas 4 e 5 apresentam os resultados da análise de sensibilidade. Observa-se que os resultados apresentam significativa variação com relação ao patamar de volatilidade de mercado, o que ocorre devido ao elevado valor tempo das opções (que são de prazo longo). Com relação à taxa de juros, observa-se que, conforme as opções entram no dinheiro (isto é, conforme as taxas de longo prazo caem em 4 pontos percentuais ao ano), tanto o valor nominal do passivo quanto sua duração e convexidade aumentam significativamente (cerca de 20 vezes). A sensibilidade na direção de elevação da taxa de juros também é significativa.

\section{Conclusões}

Este artigo apresentou um modelo para determinação do valor financeiro do passivo de companhias seguradoras atrelado às garantias concedidas aos participantes de planos PGBL e VGBL.

Propõe-se uma modelagem da opção financeira baseada em modelos derivados do modelo de Black \& Scholes, a partir da qual é possível calcular não somente o valor do passivo como também sua duração e convexidade financeiras. Essa modelagem é aplicada a uma situação real na qual observa-se que o valor total da obrigação é de $0,81 \%$ do patrimônio total do plano. No entanto, para situações em que haja uma acentuada queda da taxa de juros real,

Tabela 5. Análise de sensibilidade em relação ao nível da taxa de juros.

\begin{tabular}{cccc}
\hline $\begin{array}{c}\text { Deslocamento paralelo na } \\
\text { estrutura temporal de taxa } \\
\text { de juros (p.p.) }\end{array}$ & $\begin{array}{c}\text { Valor do passivo } \\
\text { (R\$ milhões) }\end{array}$ & $\begin{array}{c}\text { Duração financeira } \\
\text { (R\$ milhões) }\end{array}$ & $\begin{array}{c}\text { Convexidade financeira } \\
\text { (R\$ milhões) }\end{array}$ \\
\hline$+2,00$ & 1,05 & 1,15 & $-0,38$ \\
$+1,00$ & 2,07 & 2,42 & $-0,87$ \\
0,00 & 4,27 & 5,46 & $-2,19$ \\
$-1,00$ & 9,07 & 12,22 & $-5,89$ \\
$-2,00$ & 20,19 & 27,42 & $-15,09$ \\
$-3,00$ & 43,78 & 51,02 & $-21,33$ \\
$-4,00$ & 85,51 & 87,22 & $-40,38$ \\
\hline
\end{tabular}


o valor total do passivo aumentaria em cerca de 20 vezes, passando a representar cerca de 16\% do patrimônio total do plano. Não é de estranhar, portanto, que esse tipo de garantia possa gerar, no longo prazo, uma situação de elevado risco para o setor, como ocorrido no Reino Unido em finais da década de 1990 (BALLOTTA; HABERMAN, 2003).

Os modelos aqui apresentados podem ser, em pesquisas futuras, desenvolvidos em três aspectos. Pode-se ajustar o modelo para a situação em que as condições de tributação diferem em função da decisão do participante na data de aposentadoria. Além disso, pode-se ampliar o modelo para tratar da situação em que os retornos da carteira do fundo são estocásticos e correlacionados com os movimentos das taxas de juros. Por fim, o tratamento probabilístico da aposentadoria e mortalidade dos participantes representaria um refinamento atuarial para os modelos.

\section{Referências}

BALLOTTA, L.; HABERMAN, S. Valuation of guaranteed annuity conversion options. Insurance: Mathematics and Economics, v. 33, p. 87-108, 2003.

BALLOTTA, L.; HABERMAN, S. The fair valuation problem of guaranteed annuity options: the stochastic mortality environment case. Insurance: Mathematics and Economics, v. 38, p. 195-214, 2006.

BLACK, F. Interest rates as options. Journal of Finance, v. 50, p. 1371-1376, 1995.

COLEMAN, T. F.; Ll, Y.; PATRON, M. C. Hedging guarantees in variable annuities under both equity and interest rate risks. Insurance: Mathematics and Economics, v. 38, p. 215-228, 2006.

CONSIGLIO, A.; SAUNDERS, D.; ZENIOS, S. A. Asset and liability management forinsurance products with minimum guarantees: The UK case. Journal of Banking \& Finance, v. 30, p. 645-667, 2006.
DAHL, H.; MEERAUS, A.; ZENIOS, S. A. Some financial optimization models: 1 Risk management. In: ZENIOS, S. A. (Org.). Financial optimization. Cambridge: Cambridge University, 1993a.

DAHL, H.; MEERAUS, A.; ZENIOS, S. A. Some financial optimization models: 11 Financial engineering. ln: ZEN1OS, S. A. (Org.). Financial optimization. Cambridge: Cambridge University, 1993b.

FABOZZI, F. J. Fixed income readings for the chartered financial Analyst ${ }^{\circledR}$ program. New Hope: Frank J. Fabozzi Associates, 2000.

JORION, P. Value at risk: a nova fonte de referência para o controle do risco de mercado. São Paulo: Bolsa de Mercadorias \& Futuros, 1998.

KOLB, R. W. Futures, options and swaps. Malden: Blackwell Publishers, 2000.

KÜHN, C. Pricing contingent claims in incomplete markets when the holder can choose among different payoffs. Insurance: Mathematics and Economics, v. 31, p. 215-233, 2002.

LACHANCE, M. E.; MITCHELL, 0. S.; SMETTERS, K. Guaranteeing defined contribution pensions: the option to buy back a defined benefit promise. The Journal of Risk and Insurance, v. 70, n. 1, p. 1-16, 2003.

LEE, J. H.; STOCK, D. R. Embedded options and interest rate risk for insurance companies, banks and other financial institutions. The Quarterly Review of Economics and Finance, v. 40, n. 169-187, 2000.

LUEMBERGER, D. G. Investment science. Nova lorque: Oxford University Press, 1998.

MILEVSKY, M. A.; SALISBURY, T. S. Financial valuation of guaranteed minimum withdrawal benefits. Insurance: Mathematics and Economics, v. 38, p. 21-38, 2006.

SAAD, N.; RIBEIRO, C. 0. Modelos determinísticos de gestão de ativo/passivo: uma aplicação no Brasil. Revista de Contabilidade e Finanças, n. 34, p. 50-62, 2004.

SAAD, N.; RIBEIRO, C. 0. Um modelo de gestão de ativo/ passivo: aplicação para fundos de benefício definido com ativos de fluxo incerto. Revista de Contabilidade $e$ Finanças, 2005.

SCHRAGER, D. F.; PELSSER, D. F. Pricing rate of return guarantees in regular premium unit linked insurance. Insurance: Mathematics and Economics, v. 35, p. 369-398, 2004.

\title{
A model for pricing options embedded in pension products in Brazil
}

\begin{abstract}
This paper presents a model to assess liabilities and financial risks created by options embedded in retirement related investment products in Brazil (PGBL/VGBL). A specific model that characterizes the financial option embedded in insurance products is presented. These products guarantee the conversion of final participant balance in retirement income at predefined terms. Financial characteristics of this liability such as duration, convexity and equivalent cash flows are also modeled. These characteristics can be useful as inputs to optimization models for the assets portfolio backing such liabilities. Finally, results and sensitivity analysis of an application of the model to a Brazilian open-end retirement related fund are presented.
\end{abstract}

Keywords

Embedded options. Insurance. Asset/liability management. Finance. 